\title{
On the Expressive Techniques and Artistic Conception of Chinese Flute Playing Works
}

\author{
Zhinuo Tang \\ School of Music, East China Normal University, Shanghai 200042, China \\ DOI: $10.32629 /$ asc.v2i4.532
}

\begin{abstract}
This paper discusses the performance of Chinese styled works from the perspective of flute playing and the related theories of music aesthetics. As for the technique of music expression, the paper thinks that it is necessary to pay attention to the shaping of music image and the expression of music emotion to realize the unity of music expression and performance skills. In terms of the presentation of meaning and rhyme, it is necessary to highlight artistic conception in the blend of scenes, and reflect the charm through the use of embellish cavity skills and the performance of spiritual temperament.
\end{abstract}

Keywords: flute, Chinese style, music image, musical emotion, embellish cavity

\section{Introduction}

Flute is an important woodwind instrument in the family of western musical instrument, playing a very important role in solo, ensemble and other genres. Since the end of the 19th century, with the introduction of Western culture to the East and the development of the educational model of "Eastward Spread of Western Culture", flute began to be known as a part of urban music culture. Since the founding of the new China, many excellent Chinese-style music works have been produced under the pressure of teaching and performance in the context of the development of professional music education and professional music creation. From the perspective of performance, every flute player must have the ability of how to use flute to interpret Chinese style works, or how to display the aesthetic characteristics and meaning of Chinese music from the application of playing skills and musical performance. This article will talk about the author's understanding from three aspects: Flute related works with Chinese characteristics in the present situation, the technique of expression and the presentation of underlying meaning and rhyme.

\section{Current situation of music creation by flute with Chinese characteristics}

From the current situation of music creation by flute with Chinese characteristics, the embodiment of "Chinese characteristics" mainly focuses on the following three aspects: First, the adaptation of established works. Such works are mainly adapted based on established repertoire, especially folk songs and instrumental music with distinct regional and national styles. For example, "The Downward Running River" (Yunnan folk song), "Harvest in Autumn" (Northern Shaanxi folk song), "Green Willows" (folk song from Jiangsu), these works basically retain the style characteristics of the origin of folk songs with strong regional characteristics. Based on instrumental works such as "Fishing Boat in the Evening", "Flute And Drum At Sunset", "Journey to Suzhou" and so on, these works are made of different ethnic musical Instruments. In the process of adaptation, the structure, title, image, mood and other characteristics of the original song are basically retained. When using the flute for performance, on the one hand, the playing version of these songs is added; on the other hand, the transplantation and application of playing skills of national instruments is helpful to enrich the artistic performance of the flute. On the other hand, it is helpful to enrich the artistic expression of flute through the transplantation and application of playing skills of national instruments. The second is the works created according to folk music elements. These works mainly capture a certain element of folk music in the creation, or a typical tone, a typical melody motivation, a modal scale and so on. Through the combination of the use of these elements and the composer's creative thinking, the humanistic atmosphere and living customs of different regions are truly depicted and reproduced, and the characteristics of the times are also reflected. For example, the solo "The Bright Sunshine on the Tianshan Mountain" created by the famous contemporary composer Huang Huwei in the 1970s fully adopted the elements of harmony minor mode and tabla rhythm characteristic of Xinjiang ethnic minority style, taking Tianshan mountain, oasis and desert as images. The author speak highly of the beautiful mountains and rivers of the motherland and the hard-working and simple spirit of Xinjiang ethnic minorities. The third is on the diversified genre works created by using modern composition techniques. These works are mainly created 
by professional composers with the introduction of professional composition technology since the reform and opening up. These works are not limited to the solo genre, but with exploration on the integration between flute and different instruments from the perspective of Chinese music aesthetics. Such as flute and piano related works by contemporary composer Huang Anlun, flute and string quartet "Freehand brushwork in Yunnan" by Ding Yulun and so on, which basically inherited and created a western concertos, and chamber music genre characteristics, forming the form of "westernization" combined with the height of the content of "Chinese style". It reflects the development process of Chinese modern style of flute music art.

\section{Technique of expression of flute works in Chinese style}

Compared with western flute works, Chinese flute based works adhere to the aesthetic principle of traditional music in creation, that is, to pay attention to the performance of title-oriented content. Therefore, when playing these works, we need to explore the historical and cultural background of the works according to their titles, and grasp the rules of musical expression in the structure of the works. In the author's opinion, the aesthetic pursuit of Chinese style flute music can only be explored based on the image in music works and the emotion to be expressed in specific expression techniques.

\subsection{Shaping of music image}

Musical image refers to the object to be represented by music and is an important element of music content. The dialectical view of literature and art holds that the musical image is the result of the musician's aesthetic understanding of social life and aesthetic creation according to the rules of beauty. Therefore, it is "the unity of sensibility and rationality". In music creation, it reflects the composer's understanding of social life and transforms the images in life into objects that can be molded. In musical performance, it reflects the performer's understanding of musical images and shapes them through sound techniques. In flute playing, the musical image is determined according to the title of the work, and then the image is shaped, which is the most basic interpretation path of Chinese style works.

Taking Dai Hongwei's work of the On the Grassland of Inner Mongolia as an example, this piece is a flute solo based on a single theme. On the whole, it is divided into four parts: introduction, middle board, allegro and epilogue. According to the characteristics of theme presentation and change, there are basically two kinds of images: One is the description and singing melody with long tone elements as the first theme, mainly depicting the natural scene of blue sky, white clouds and lush water grass in Inner Mongolia grassland. The other is the combination of rhythmic sound patterns and lyric phrases to form a passionate scene of horse racing and dancing, which belongs to the category of rich humanistic scenes. In shaping these two images, it is necessary to match the characteristics of the image with the coordination of Qi, finger and tongue. For example, in the performance of the introduction part, through the breath control function of abdominal muscles and diaphragm, the image of the vast and boundless grassland is displayed with different dynamics and hierarchical changes according to the characteristics of the melody. The change of sound levels at different heights gives people a visual sense of synesthesia, as if you are in a grassland full of spectacular scenery through the sound. On the performance of allegro, it combines the stopping and breaking of breath with the rich and jumping sound, showing the scene of grassland horse racing. Among them, in the use of pronunciation, we need to pay attention to the use of the tip of the tongue spit method, spit the power of playing on a point, to be full of explosive force and sense of life, in the combination of lyric sentences through the coherence of breath technology to achieve the beauty of point, line combination of sound.

\subsection{Expression of musical emotion}

In view of the title characteristic of Chinese style flute works, there is not only special indication in the expression of music image, but also internal stipulation in the expression of music emotion. In terms of the expression of musical emotion, it should be realized through the three organic connection processes of emotion, emotion and expression. "Li Qing" refers to the development of plots in Li Tong music, and the specific emotion direction to be expressed by composers can be identified according to different structures. Li Yu, a drama theorist in the Qing Dynasty, said in his monograph "Xian Qing Note " that "songs should be sung with emotions, and those who are sung with emotions should also have the plots in the songs". Therefore, the analysis of the plot structure is the first step to achieve emotional expression. "Harmony" refers to that in the performance process, the performer should integrate his own emotions into the work and form emotional resonance with the music sound, so as to express the true feelings to be expressed by the music. "Expression" is to externalize inner emotions into performance. Only when a body language and a look can reveal inner emotions, can the standard of "performance" be achieved.

Taking Li Guoquan's flute work "Fishing Boat in the Evening" as an example, this piece is based on the ancient music material and integrates the timbre characteristics of different sound areas of flute, and uses its unique linear melody form to visually reproduce a leisurely and carefree landscape painting axis. In light of the expression of emotion in this song, 
we should think about the relationship between scene and emotion. The so-called "scenery", in fact, is to fit the title of the music, "fishing", "boat", "singing", "evening" are four different scenes, the song is also the four scenes as the expression object, so as to sublimate the yearning for leisure and comfortable life admiration and pursuit of feeling. Music in middle part as an example, this part to five level into the scale type melody and octave upper and lower into the structure of the form a psychological emotional space, show the intrinsic relationships between reality and ideal, the present and future, need when playing with the combination of tongue and liaison and strength will change two "situation" there are both opposite and unified relationship, In the performance of allegro part, through the combination of prominent rhythm stress and leaping articulation, a surging passion is displayed in the melody of The model jin. It indicates the pursuit of poetic life and the exploration of eternal vitality.

\section{The presentation of the underlying meaning and charm of Chinese flute works from the aesthetic point of view}

From the current Chinese style flute music creation and expression technique, reflects the aesthetic characteristics of traditional Chinese music, both in creation and performance, they attach great importance to the presentation of meaning and rhyme. "Underlying meaning" is a compound vocabulary of artistic conception and lasting appeal in the aesthetic category of traditional Chinese music. The word "artistic conception", in a nutshell, refers to the "scene in love, love in the scene, scene blend". In the Chinese-style flute works, the works with scenery as the expression object for emotional expression can be said to occupy the majority of the weight, which is mainly reflected in the title of the work. From the perspective of performance, the presentation of artistic conception is, in fact, to always highlight a sense of painting in performance, to construct sound through the depiction of the picture, and then to construct the picture through the coordination of Qi, finger, tongue and other skills. In the expression of emotion, it is necessary to fully grasp the theme connotation of the work, through the analysis of the creation background of the work directly to the theme implied by the sound, to provide specific emotional categories for the performer, and then to integrate their feelings and thoughts into the performance, combined with the specific picture. Such as "On the Inner Mongolia Grassland", "Sing on Fishing Boat at Night" and other works are to highlight the depiction of the scene in the way of picture presentation, and then in the scenery to show the praise of the motherland and the pursuit of a better life, From scene sublimation to sentiment, from sentiment externalization and scenery, the combination of the two can express the performance of artistic conception.

From the performance of lasting appeal, it means that the performance should highlight the regional or national style, and show the specific humanistic feelings and spiritual temperament. The author thinks that, on the specific music performance, First of all, we should pay attention to the use of embellish cavity skills. Runqiang is a concept put forward by Yu Huiyong, a contemporary ethnomusicologist. Colloquially speaking, it is the technique of embellishing melody, such as in Inner Mongolia Grassland, the use of grace notes, in fact, such as Boeing, appoggiatura and vibrato, highly imitates the singing skills of twists and turns in long-tone folk songs. Therefore, when playing this song, the understanding of embellishing technique of long-tone folk songs and the combination of grace notes in the music will help to enhance the performance of Mongolian style charm. The second is to pay attention to the performance of spiritual temperament, which means to grasp the connotation of music in the analysis and performance of music. For example, in the song "Sing on Fishing boat at Night", behind the relaxed feeling expressed in the music, in fact, it wants to express a spirit of harmony between nature and man, namely the connotation of harmonious coexistence between nature and man.

\section{Conclusion}

Modern literary and art theory believes that style is the style that is reflected from interior content, which means that the creation of literature and art should be based on specific aesthetic requirements, including the creation of ideas, genres, themes and materials, and other elements should be based on certain national characteristics and times. The so-called " to reflect external expression" means that the presentation of works should have a certain "character", reflecting the characteristics of specific artistic materials under the aesthetic connotation. In terms of "Chinese style" in music vision, it refers to the use of unique Chinese musical elements and aesthetic pursuit in music creation, and the unique spirit of the Chinese nation and humanistic connotation can be reflected in the performance, singing and other practices. Just as Huang $\mathrm{Zi}$, a famous modern composer and educator, said, "The art of an era reflects the life of an era, and all great art can be regarded as the portrayal of a nation and society. " As a flutist, when interpreting Chinese-style works, he should pay attention to the style of music, and comprehensively consider his performance from the Angle of music understanding and playing skills, so as to "Ascend another storey to have a further sight retrospect and prospect". 


\section{References}

[1] Luo Yifeng. Research on the history of Chinese music thought on the outlook on history, material and method[J]. Music of China, 2021 (9).

[2] Wei Wei, Wei Huang. Some basic problems in flute performance and teaching[J]. Journal of Tianjin Conservatory of Music, 2018 (9).

[3] Lu Jialiang. Analysis of cultural Aesthetics in the Transplantation of Flute Song "Flute and Drum at Sunset"[J]. Musical Works, 2018 (6). 